\title{
Phosphodiesterase Type 4 Inhibition in CNS Diseases
}

Citation for published version (APA):

Blokland, A., Heckman, P., Vanmierlo, T., Schreiber, R., Paes, D., \& Prickaerts, J. (2019).

Phosphodiesterase Type 4 Inhibition in CNS Diseases. Trends in Pharmacological Sciences, 40(12), 971985. https://doi.org/10.1016/j.tips.2019.10.006

Document status and date:

Published: 01/12/2019

DOI:

10.1016/j.tips.2019.10.006

Document Version:

Publisher's PDF, also known as Version of record

Document license:

Taverne

\section{Please check the document version of this publication:}

- A submitted manuscript is the version of the article upon submission and before peer-review. There can be important differences between the submitted version and the official published version of record.

People interested in the research are advised to contact the author for the final version of the publication, or visit the DOI to the publisher's website.

- The final author version and the galley proof are versions of the publication after peer review.

- The final published version features the final layout of the paper including the volume, issue and page numbers.

Link to publication

\footnotetext{
General rights rights.

- You may freely distribute the URL identifying the publication in the public portal. please follow below link for the End User Agreement:

www.umlib.nl/taverne-license

Take down policy

If you believe that this document breaches copyright please contact us at:

repository@maastrichtuniversity.nl

providing details and we will investigate your claim.
}

Copyright and moral rights for the publications made accessible in the public portal are retained by the authors and/or other copyright owners and it is a condition of accessing publications that users recognise and abide by the legal requirements associated with these

- Users may download and print one copy of any publication from the public portal for the purpose of private study or research.

- You may not further distribute the material or use it for any profit-making activity or commercial gain

If the publication is distributed under the terms of Article $25 \mathrm{fa}$ of the Dutch Copyright Act, indicated by the "Taverne" license above, 


\title{
Phosphodiesterase Type 4 Inhibition in CNS Diseases
}

\author{
Arjan Blokland, ${ }^{1, \star}$ Pim Heckman, ${ }^{2}$ Tim Vanmierlo, ${ }^{3,4}$ Rudy Schreiber, ${ }^{1}$ Dean Paes, ${ }^{3,4}$ and Jos Prickaerts ${ }^{4}$
}

\begin{abstract}
Phosphodiesterases (PDEs) have been an interesting drug target for many diseases. Although a vast number of mainly preclinical studies demonstrates beneficial effects of PDE inhibitors for central nervous system (CNS) diseases, no drugs are currently available for CNS indications. In this review, we discuss the rationale of PDE4 inhibitors for different CNS diseases, including memory impairments, striatal disorders, multiple sclerosis (MS), and acquired brain injury (ABI). However, clinical development has been problematic due to mechanism-based adverse effects of these drugs in humans. Our increased understanding of factors influencing the conformational state of the PDE4 enzyme and of how to influence the binding affinity of PDE4 subtype inhibitors, holds promise for the successful development of novel selective PDE4 inhibitors with higher efficacy and fewer adverse effects.
\end{abstract}

\section{PDE Inhibitors: Basic Properties and Current Status}

PDEs were first discovered $\sim 50$ years ago and have attracted much attention in various research fields [1]. Based on their regulation of intracellular cAMP and cGMP levels, PDEs have a pivotal role in cellular functions. Not surprisingly, there has been significant interest in how PDEs regulate cell function and whether their activity can be modulated to treat diseases. One of the first studies reporting the role of PDEs in the regulation of intracellular CAMP signaling in the kidney was published in 1968 [2]. Some years later, a paper was published that described the effects of xanthine derivatives as inhibitors of PDE enzyme activity in fat cells [3]. In 1972, the first evidence was found for two different types of PDE in amoebas. Since then, more subtypes have been described, with 11 mammalian PDE families (PDE1-PDE11) currently described [1,4].

These families are categorized based on features such as mechanisms of regulation, subcellular distributions, and enzymatic and kinetic properties. In addition, each family contains multiple subtypes and/or genes (e.g., PDE1A and PDE4B), which can encode several transcript variants (e.g., PDE4D1PDE4D9). Currently, this results in >100 PDE types, sometimes referred to as the 'PDE superfamily'. PDEs occur in many cell types throughout the body and exert their functions by regulating the cyclic nucleotides cGMP and cAMP. Of note, PDE families differ in their ability to bind and degrade substrates, which can be cAMP selective, cGMP selective, or both (reviewed in [1]).

PDE gene families are also expressed in an organ-specific manner (e.g., [5]). Understanding the distribution of PDEs in the body and brain has been essential for selecting new drug targets for PDE inhibitors for different diseases [6]. For example, the localization of PDE4 in inflammatory cells (keratinocytes, neutrophils, and T cells) led to the development of PDE inhibitors for clinical use in chronic obstructive pulmonary disease (COPD), atopic dermatitis, and psoriasis (e.g., [7,8]). In addition, selective PDE inhibitors have been developed and approved for treating cardiovascular and intermittent claudication (e.g., $[9,10])$. These applications indicate that PDE inhibitors have clear clinical potential. Although there has much research focusing on developing PDE inhibitors for CNS disorders, there are no PDE drugs currently approved for clinical use in this field (e.g., [11]). Various reasons have been offered for the failures in the clinical development of selective PDE inhibitors in CNS diseases [12,13].

Issues impacting the clinical efficacy of PDE inhibitors in CNS diseases might also be related to a lack of knowledge regarding their precise role in intracellular signaling pathways. Although PDE inhibitors are generally known to degrade CGMP and CAMP, the actual effects of PDEs and their inhibitors on overall cell physiology appear to be more complex (e.g., $[1,14-16])$. For example, inhibition of PDE1 in striatal medium spiny neurons (MSNs) decreased the level of surface AMPA receptors, which are
Highlights

Many preclinical studies show positive effects of PDE inhibitors in CNS disease models. However, there are no clinically approved PDE inhibitors for CNS indications.

For PDE4, approval has been hampered mainly because of adverse effects associated with the inhibitors.

PDE4 inhibition appears to be effective for many CNS indications, given that it has shown beneficial effects on memory in older healthy volunteers and patients with schizophrenia.

PDE4 inhibition can lead to improved neuronal plasticity and promote antineuroinflammatory effects. These effects underlie restorative effects in models of memory impairment, schizophrenia, attention-deficit hyperactivity disorder, MS, and $\mathrm{ABI}$

Understanding the factors influencing the conformational state of the PDE4 enzyme, and how to influence the binding affinity of PDE4 subtype inhibitors, holds promise for the successful development of novel selective PDE4 inhibitors with a desirable therapeutic window.

1Department of Neuropsychology and Psychopharmacology, EURON, Faculty of Psychology and Neuroscience, Maastricht University, Maastricht, The Netherlands

${ }^{2}$ Neurobiology Expertise Group, Groningen Institute for Evolutionary Life Sciences (GELIFES), Faculty of Science and Engineering (FSE), University of Groningen, Groningen, The Netherlands ${ }^{3}$ Department of Immunology and Biochemistry, Biomedical Research Institute, Hasselt University, Hasselt, Belgium 
regulated by the allosteric activation of PDE2 [17]. This complex interactive regulation of cellular processes is related to the compartmentalization of specific PDEs. Given this complex regulation of intracellular signaling by PDEs, and the apparent unique profile and function of the different gene families and isoforms, a thorough understanding of these processes will be required to successfully develop selective drugs.

The expression of different PDEs in the brain is relevant for selecting PDE targets for specific brain diseases. However, the expression of PDEs can be delineated at different levels. The first is the expression pattern at the gene level in different brain structures. Lakics et al. showed that the expression of PDE gene families in the brain and periphery was heterogeneous [5]. These data hint at PDE subtypes that could be appropriate targets for drugs to treat CNS diseases based on expression in disease-relevant brain structures. However, these data only show a global expression level. Only a relatively limited number of studies have used PDE-selective antibodies to investigate their subcellular localization in neurons and their role in signaling pathways (e.g., [18]), and single cell RNAsequencing studies usually do not distinguish between transcripts encoding different PDE isoforms (e.g., [19]). This limits our understanding of the cellular functions of PDEs (e.g., [20]), and how compartmentalized PDE signaling might lead to altered brain function.

Although more research is needed to understand the complex regulation of cellular processes by PDEs, many animal studies show beneficial effects of selective PDE inhibitors in preclinical models of CNS diseases. Table 1 provides a global overview of these studies listing different disease categories in relation to their PDE subtype (for a general overview. see [1]). For example, there is support for PDE1 inhibitors for Alzheimer's disease (AD) and schizophrenia [21-24], whereas PDE2 inhibitors were shown to be active in animal models for memory dysfunction [23] and some studies hinted at an antidepressant effect [25]. For PDE3 inhibition, there is strong evidence that it could have beneficial effects in stroke $[26,27]$ and, to a lesser extent, in animal models of memory dysfunction $[11,28]$. PDE4 inhibitors have been shown to be effective in different disease areas, such as stroke [26,29], animal models of AD [11,30], models of schizophrenia [21,31], MS [32,33], and different developmental disorders [34-37]. Animal studies also showed antidepressant effects after PDE4 inhibition [30,38]. Interestingly, studies in humans have shown positive effects of PDE4 inhibition on cognition in healthy older subjects [39] and patients with schizophrenia [40]. For PDE5, there is some preclinical evidence for a role in cognition models [23] and in stroke [23,26], whereas human studies showed an indication for memory-enhancing effects [41] but no effects on cognition [42].

For PDE7 inhibitors, some effects on cognition have been found, but the most promising data have been shown in models of MS [33]. Some preclinical studies showed promising effects of PDE9 inhibitors in cognition models $[11,43]$, but failed to improve cognitive performance in schizophrenic patients [44]. PDE10 inhibitors have been developed for treating corticostriatal disorders, including schizophrenia [45-47], but clinical studies in schizophrenia have been disappointing. For PDE11, only a few animal studies show relevance for improved social memory [48].

This overview strongly supports the notion that PDE inhibition is beneficial for treating different CNS diseases. PDE4 appears to be an attractive molecular target for several reasons. First, it is strongly expressed in brain regions and neurons and/or cells related to these different disorders (e.g., $[5,49])$. Second, preclinical data with PDE4 inhibitors show positive effects in different disease areas (Table 1). Third, beneficial effects of PDE4 inhibition can be linked to signaling pathways underlying neuroplasticity and inflammation $[49,50]$ and, fourth, some clinical studies showed positive effects on memory in healthy older subjects and in patients with schizophrenia $[39,40]$. Therefore, here, we highlight the potential of PDE4 inhibitors in different CNS diseases.

\section{PDE4 and Memory}

There is strong evidence for a role of PDE4 in memory formation, largely based on seminal work by Eric Kandel on the molecular mechanisms of memory [50]. In this framework, cAMP is an essential second messenger that leads to activation of protein kinase $A$ (PKA) and, subsequently, the
${ }^{4}$ Department of Psychiatry \& Neuropsychology, School for Mental Health and Neuroscience, EURON Maastricht University, Maastricht, The Netherlands

*Correspondence: a.blokland@maastrichtuniversity.nl 
Table 1. Overview of the different PDE families and their possible relevance for different CNS disease $\operatorname{areas}^{a, b, c}$

\begin{tabular}{|c|c|c|}
\hline PDE family & CNS disease area & Experimental support \\
\hline \multirow[t]{2}{*}{ PDE1 } & AD/MCI (cognition) & $x X$ \\
\hline & Schizophrenia & $X X$ \\
\hline \multirow[t]{2}{*}{ PDE2 } & AD/MCI (cognition) & $X X$ \\
\hline & Depression & $x$ \\
\hline \multirow[t]{2}{*}{ PDE3 } & Stroke & $X X X$ \\
\hline & AD/MCl (cognition) & $X X$ \\
\hline \multirow[t]{6}{*}{ PDE4 } & Stroke & $X X X$ \\
\hline & AD/MCl (cognition) & $\mathrm{XXX}, \mathrm{H}+$ \\
\hline & Schizophrenia (cognition) & $\mathrm{XXX}, \mathrm{H}+$ \\
\hline & Depression & $X X$ \\
\hline & Multiple sclerosis & $X X X$ \\
\hline & Developmental disorders & $X X X$ \\
\hline \multirow[t]{2}{*}{ PDE5 } & Stroke & $X X$ \\
\hline & AD/MCl (cognition) & $X X, H+/-$ \\
\hline \multirow[t]{2}{*}{ PDE7 } & AD/MCl (cognition) & $x$ \\
\hline & Multiple sclerosis & $X X$ \\
\hline PDE9 & AD/MCl (cognition) & $\mathrm{XX}, \mathrm{H}-$ \\
\hline \multirow[t]{3}{*}{ PDE10 } & $\begin{array}{l}\text { Huntington's and } \\
\text { Parkinson's disease }\end{array}$ & $X X X$ \\
\hline & Schizophrenia & $\mathrm{XXX}, \mathrm{H}_{-}$ \\
\hline & AD/MCl (cognition) & $X X$ \\
\hline PDE11 & $\begin{array}{l}\text { Not disease specific (social } \\
\text { memory) }\end{array}$ & $x$ \\
\hline
\end{tabular}

a Abbreviations: $\mathrm{AD}$, Alzheimer's disease; $\mathrm{MCl}$, mild cognitive impairment.

${ }^{b}$ Experimental support is rated on basis of preclinical studies $[X$ (marginal)-XXX (strong)] and human studies $(H+$, positive findings in humans; $\mathrm{H}$-, negative findings in humans).

'PDE6 is only expressed in photoreceptors and pineal gland and, therefore, is not included. PDE8 is also not included because too few data are available.

phosphorylation of cAMP response element-binding protein (CREB $\rightarrow$ pCREB; Figure 1). PKA activation also leads to insertion of AMPA receptors into the pre-synaptic membrane [51]. pCREB is responsible for the transcription of neuronal plasticity genes, including those encoding AMPA receptors and brain-derived neurotrophic factor (BDNF) $[29,52]$. Linked to this, CAMP has been found to have a pivotal role in the induction and maintenance of long-term potentiation (LTP) [50]. Given that PDE4 is located in hippocampal neurons and shows specificity towards CAMP, inhibition of PDE4 can elevate cAMP levels and improve LTP [53]. Consequently, PDE4 is important for hippocampal functions via: (i) presynaptically enhancing glutamate (and also acetylcholine) synthesis and release; and (ii) postsynaptically by stimulating neurotransmitter(s) receptor signaling. In line with these notions, the nonselective PDE4 inhibitor rolipram was shown to improve memory in rodents. This finding has been replicated in many other animal models and with more (subtype) selective PDE4 inhibitors [54]. 


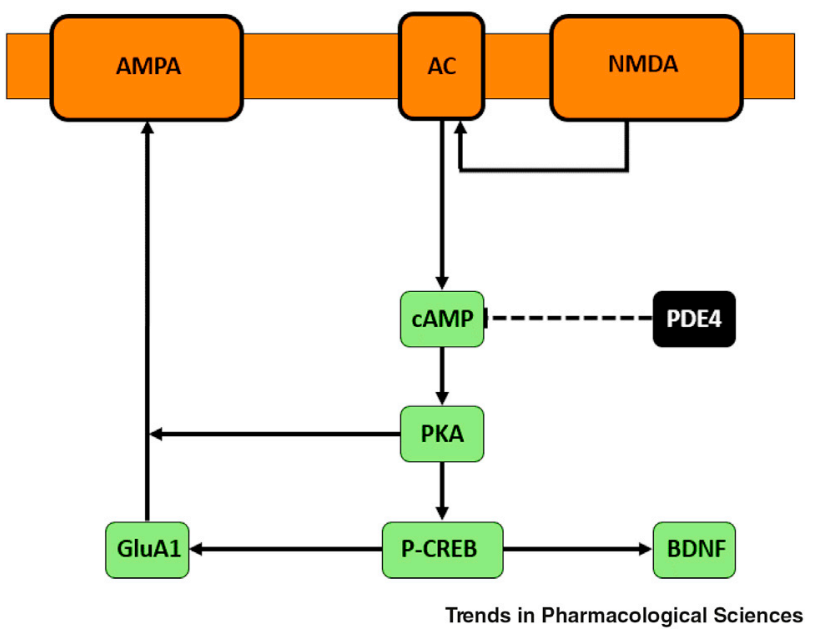

Figure 1. Phosphodiesterase 4 (PDE4) in Hippocampal Neurons.

Within the hippocampus, signal transduction is achieved through activation of glutamate NMDA receptors on the postsynaptic membrane of neurons, through which $\mathrm{Ca}^{2+}$ subsequently enters the cell. In this way, glutamate induces long-term potentiation (LTP), which is the underlying physiological substrate of learning and memory. LTP is maintained by the insertion of more AMPA receptors into the postsynaptic membrane. $\mathrm{Ca}^{2}+$ binds to calmodulin (CaM) and this complex activates $\mathrm{Ca}^{2+} /$ calmodulin kinase (CaMK), which can stimulate the insertion of AMPA receptors into the membrane. In addition, CaMK can activate adenylate cyclase (AC) and this leads to subsequent production of CAMP, which activates PKA. Next, protein kinase A (PKA) phosphorylates the GluA1 subunit of the AMPA receptor, thus also promoting the trafficking and insertion of already existing AMPA receptors into the membrane [71]. Finally, PKA also phosphorylates the transcription factor CAMP response element-binding protein (CREB), which is a crucial step in protein transcription, including that of AMPA receptors and brain-derived neurotrophic factor (BDNF). Thus, more AMPA receptors become available for trafficking and insertion into the membrane. Unbroken lines with arrows represent excitatory connections; broken lines with blunted arrowheads represent an inhibitory connection.

Thus, PDE4 inhibition appears to have a strong straightforward rationale, and different drug discovery programs have aimed to develop a PDE4 inhibitor to treat memory disorders [11]. However, PDE4 inhibitors have been associated with severe adverse effects, mainly emesis. This has been related to the expression of PDE4D in regions related to the emetic response [55]. Recently, PDE4 subtype-selective inhibitors were developed to maximize the therapeutic window and minimize adverse effects. These data suggest that PDE4D is more relevant for cognition enhancement (e.g., [56]). By contrast, recent studies showed that a nonselective PDE4 inhibitor (roflumilast) had beneficial effects on memory in humans without any clear adverse effects $[39,40]$. Thus, understanding this beneficial effect of roflumilast could open new avenues for developing PDE4 inhibitors to improve memory performance.

\section{PDE4 and Corticostriatal Functions}

MSNs are the main neuronal cells in striatum; although they receive glutamatergic projections from the cortex, their plasticity is dependent on dopaminergic signaling [57]. They are the only projection neurons of the striatum, integrating all input to this brain region (e.g., [13]). Most of the information arriving at striatal MSNs is conveyed via cyclic nucleotide pathways, with a major role for cAMP (Figure 2). Signal compartmentalization is achieved via the generation of cyclic nucleotide compartments by PDEs, with a prominent role for PDE4 $[4,13,58]$. In analogy to its hippocampal functions, PDE4 exerts its corticostriatal functions via two mechanisms of action: (i) presynaptically enhancing dopamine synthesis, release, and metabolism, as well as dopamine D1 receptor signaling; and (ii) postsynaptically stimulating and/or inhibiting dopamine receptor signaling. Both functions independently constitute rationales for how PDE4 can regulate corticostriatal functions. 


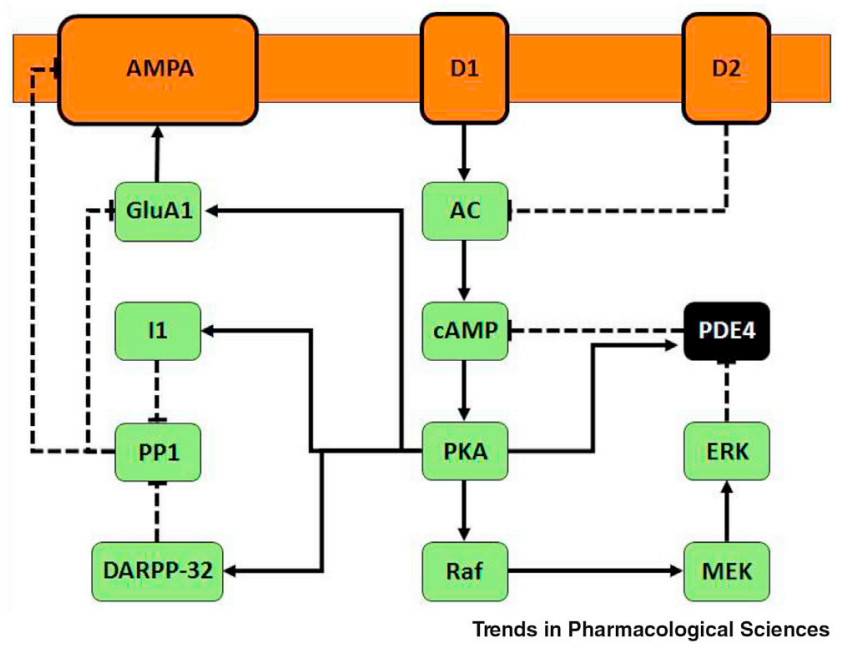

Figure 2. Phosphodiesterase 4 (PDE4) in Medium Spiny Neurons (MSNs).

Within the striatum, signal transduction (cell excitability) is achieved through activation of glutamate receptors on MSNs. However, experience-dependent modulation of synaptic strength (learning) requires dopamine. This is in contrast to other central synapses, where glutamatergic input and intracellular $\mathrm{Ca}^{2+}$ levels determine the direction and/or magnitude of synaptic plasticity. Thus, dopamine affects both ongoing behavior (cell excitability) and future behavior (learning) through its activation and inhibition of striatal cAMP. In turn, PDE4 is a main contributor to the spatial and temporal dynamics of CAMP after a cortical signal enters the striatum. As a result, PDE4 is also a main regulator of both signal transduction and plasticity. Interestingly, because of its differential expression in striatal MSNs of the direct and indirect pathway, PDE4 exerts its effect more strongly through the indirect pathway. This has both physiological and clinical implications. The striatal pathways are also characterized by their differences in dopamine receptor expression. Direct pathway dopamine D1receptors stimulate cAMP production, whereas indirect pathway dopamine D2-receptors inhibit cAMP. Finally, PDE4 itself is also regulated through different mechanisms. Dopamine-activated protein kinase A (PKA) stimulates long PDE4 isoforms and serves as a long-term inhibitory feedback mechanism. Conversely, long PDE4 isoforms are inhibited by extracellular receptor kinase (ERK), which itself is activated by PKA through rapidly accelerated fibrosarcoma (Raf) and MAPK ERK kinase (MEK). Thus, both PKA and ERK control PDE4 activity and striatal output as a key step in dopamine-induced striatal neuroplasticity. In turn, PDE4 regulates cell excitability and corticostriatal neuroplasticity through its regulation of PKA/dopamine- and CAMP-regulated phosphoprotein $32 \mathrm{kDa}$ (DARPP-32), which can ultimately affect processes such as AMPA receptor trafficking and membrane insertion. Unbroken lines with arrows represent excitatory connections; broken lines with blunted arrow heads represent inhibitory connections. Abbreviations: AC, adenylate cyclase; GluA1, AMPA receptor subunit; 11, inhibitor 1 (PP1 inhibitor); PP1, protein phosphatase 1.

Regarding its presynaptic effects, PDE4 is expressed at dopaminergic terminals in neurons of the substantia nigra pars compacta (SNc), where its inhibition leads to enhanced dopamine release [59]. By increasing the levels of dopamine at corticostriatal synapses, PDE4 inhibitors could have therapeutic potential for disorders characterized by corticostriatal hypodopaminergia, including attention deficit hyperactivity disorder (ADHD) and Parkinson's disease (PD). Additionally, it has been described that PDE4B is localized at DARPP-32-expressing neurons in the mouse frontal cortex [31]. Here, rolipram enhanced dopamine D1 receptor-induced phosphorylation of DARPP-32. Thus, this presynaptic regulation of dopamine release and enhancement of dopamine receptor signaling provides an interesting scientific rationale for PDE4 as a molecular target for novel therapeutics of disorders that involve corticostriatal hypodopaminergia (Figure 3).

The second main rationale for PDE4 in corticostriatal functions is linked to its regulation of postsynaptic dopamine receptor signaling in MSNs of both the direct and indirect pathway, as shown in striatal slices and in vivo [31]. Downstream of cAMP, the PDE4 inhibitor rolipram increased the phosphorylation of DARPP-32 and enhanced adenosine $A_{2 a}$ receptor-mediated phosphorylation of 


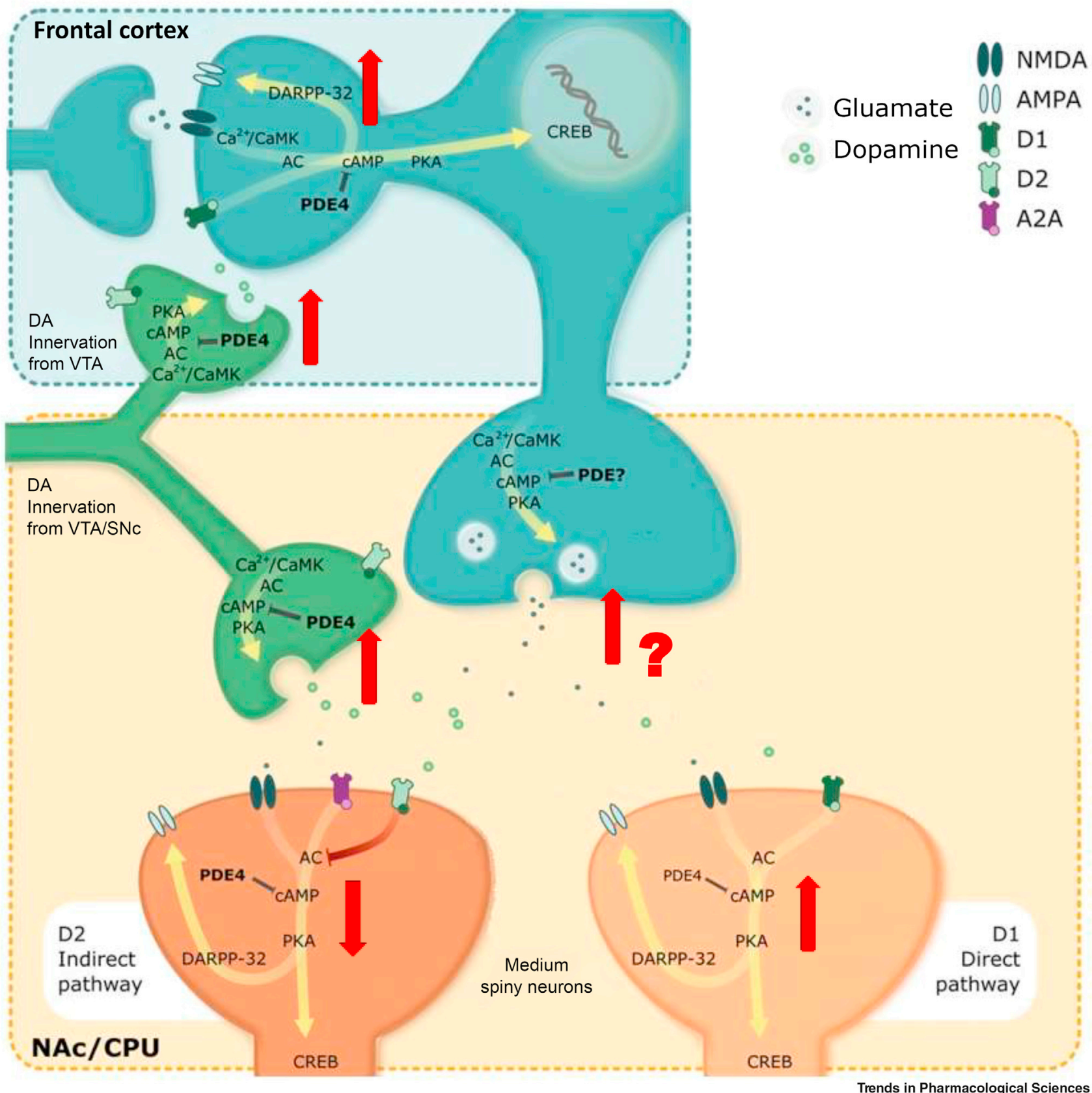

Figure 3. Role of Phosphodiesterase 4 (PDE4) in the Corticostriatal Network.

At corticostriatal synapses, the effect of PDE4 inhibition on cAMP/protein kinase A (PKA) signaling is linked to indirect pathway adenosine A2A receptor signaling and has no major role in dopamine (DA) D1-receptor direct pathway signaling. The opposite situation is observed in frontal dopaminergic signaling. In the frontal cortex, PDE4 is localized at dopamine- and cAMP-regulated phosphoprotein $32 \mathrm{kDa}$ (DARPP-32)-expressing neurons. In contrast to the striatum, PDE4 inhibition enhances dopamine D1-receptor-induced phosphorylation of DARPP-32 in the frontal cortex, indicating a prominent role of PDE4 in frontal dopamine receptor signaling. Finally, dopamine release from dopaminergic midbrain terminals can be influenced by a PDE4 inhibitor because dopamine is expressed at dopaminergic terminals in neurons of the substantia nigra pars compacta (SNc) in which cAMP has been reported to be a strong inducer of tyrosine hydroxylase gene transcription rate and mRNA, affecting dopamine synthesis and release. Upward pointing red arrows indicate stimulatory effects (behavioral activation) benefitting disorders characterized by hypodopaminergia, including attention-deficit hyperactivity disorder (ADHD) and Parkinson's disease. The downward-pointing arrow indicates inhibitory effects (behavioral inhibition) potentially 
DARPP-32 (representative of indirect pathway activation). Conversely, rolipram did not affect dopamine D1 receptor-mediated phosphorylation of DARPP-32 (representative of direct pathway activation). These findings suggest that PDE4 is exclusively expressed in indirect pathway MSNs. Immunohistochemical analysis of striatal slices revealed that PDE4B expression can be found in both pathways but with higher expression in MSNs of the indirect pathway [31]. Given this main indirect pathway activation, PDE4 inhibitors are considered as a symptomatic treatment for hyperkinetic movement disorders (e.g., Huntington's disease, HD). This is further supported by data from HD mouse models that showed increased expression of PDE4B in striatum and cortex [60]. This increase in PDE4 activity appears to be driven by mutant Huntingtin sequestering DISC1, a protein that would normally bind to and inhibit PDE4B.

Activation of the inhibitory indirect pathway by PDE4 inhibitors also mimics the action of dopamine D2 receptor antagonists, known for their antipsychotic potential. As a result, PDE4 inhibitors have been investigated as a treatment for positive symptoms in schizophrenia. Additionally, PDE4 inhibition has proven to benefit cognitive function in clinical studies and preclinical models of schizophrenia [13]. The involvement of PDE4 in schizophrenia is further supported by the interaction of PDE4B with DISC1, because a chromosomal translocation of this gene increases susceptibility for schizophrenia by disrupting binding of DISC1 to PDE4B [31]. Recent studies in humans supported the idea that PDE4 inhibition could have beneficial effects in schizophrenia [40].

Although not extensively investigated, PDE4 might prove a therapeutic target in different diseases related to other disturbed corticostriatal functions (Figure 3). Addiction and obsessive compulsive disorder (OCD) are examples of diseases in which PDE inhibition could be effective. Using behavioral sensitization, conditioned place preference, and drug self-administration as behavioral models, various studies have shown that local or systemic administration of PDE4 inhibitors reduced drug intake and/or drug seeking for psychostimulants, alcohol, and opioids in rats or mice [61]. In patients with $O C D$, activation of the indirect pathway could result in similar behavioral inhibition.

\section{PDE4 and Multiple Sclerosis}

As mentioned earlier, PDE4 inhibitors are being used to treat inflammatory diseases such as COPD and psoriasis. The fact that neuroinflammation is also a hallmark of MS provides a good rationale to explore the therapeutic potential of selective PDE inhibitors against this disease [62]. The cellular pathogenesis of MS is driven by perivenular infiltration of autoreactive lymphocytes that creates a proinflammatory microenvironment triggering phagocyte-induced CNS damage.

CAMP has three important functions in inflammation: (i) it decreases endothelial junctional permeability at the level of the blood-brain barrier (BBB) and diminishes transendothelial transport of inflammatory mediators [26]; (ii) it drives the development of regulatory $T$ cells (Tregs) to maintain immunological homeostasis [63]; and (iii) it differentiates phagocytes into an anti-inflammatory, repair-inducing phenotype [64]. The role of PDE4 has been studied for all three mechanisms. First, PDE4 (and PDE7) inhibitors were found to reduce cerebrovascular endothelial permeability in experimental autoimmune encephalomyelitis (EAE), a neuroinflammatory animal model of MS [62]. Second, inhibition of PDE4 decreased T cell proliferation and reduced the secretion of proinflammatory cytokines (TNF- $\alpha$ and IL-17), while increasing the release of anti-inflammatory cytokines (IL-10) in EAE mice [62]. Interestingly, upon anti-CD3/CD28 stimulation of primary human CD4+ naive or memory $T$ cells, the enzymatic activities of PDE4A and PDE4D alone were upregulated, although mRNA levels of PDE4A, PDE4B, and PDE4D were increased [65]. Furthermore, knockdown of all PDE4 subtypes in these activated human $C D 4+T$ cells with small interfering (si)RNA reduced their proliferation rate and inhibited the secretion of IFN- $\gamma$, revealing a primary role for PDE4D in inflammation [62]. Based on

benefitting disorders characterized by behavioral excess, such as Huntington's disease, schizophrenia, obsessive-compulsive disorder (OCD), and addiction. Whether PDE4 inhibition leads to increased glutamate release in corticostriatal neurons remains unclear. Abbreviations: AC, adenylate cyclase; CaMK, $\mathrm{Ca}^{2+} /$ calmodulin kinase; CPU, caudate putamen complex; CREB, cAMP response element-binding protein; NAc, nucleus accumbens; VTA, ventral tegmental area. 
these findings, cAMP-specific PDE inhibition in T cells can decrease inflammatory cytokine production by acting directly on Th1 and Th17 cells or by regulating the immune response through activation of Tregs.

A third role for CAMP in the control of the inflammatory process in MS involves modulating phagocyte function in the CNS. CNS-infiltrating and resident phagocytes contribute to the inflammatory response by producing proinflammatory cytokines and chemokines while triggering demyelination [66]. Increasing cAMP skews phagocytes towards an anti-inflammatory phenotype characterized by high levels of arginase 1 (Arg1), thereby hampering phagocytosis [67]. In line with this, inhibition of PDE4 was found to shift the inflammatory response in different models towards an anti-inflammatory response (e.g., [68]).

Although promising results were obtained in preclinical studies, no definitive positive clinical proofof-concept data with PDE4 inhibitors in patients with MS have been published. Results from a recent clinical trial with the nonselective PDE4 inhibitor ibudilast appear promising. This drug did not reduce focal inflammatory activity in relapsing MS, but did attenuate MS-related brain atrophy $[69,70]$. These findings indicate that PDE4 inhibition might not be relevant for relapsing MS, but might be suitable for treatment of progressive MS phenotypes. For future research, identification of the key PDE4 genes and isoforms involved in specific disease phases and processes could lead to the development of more effective and better tolerated PDE4 isoform-selective inhibitors for the treatment of MS.

\section{PDE4 in Acquired Brain Injury}

Acute brain trauma (nontraumatic, such as stroke, and traumatic, such as accidents) causes ruptured microvessels, which lead to secondary pathophysiological processes, including inflammation, cellular stress, and activation of apoptotic cascades. These in turn can result in myriad subacute and chronic effects at the molecular, cellular, subcellular, and brain function level (Figure 4). Certain changes occur rapidly, whereas others can last for many months after the lesion [71]. The BBB has a central role in the pathophysiology of $A B I$. The sustained increase in $B B B$ permeability and the subsequent leakage of inflammatory cells and humoral factors can lead to long-lasting impairments in BBB integrity [72]

The role of CAMP and PDE4 during this postinjury increase in the permeability of the endothelial cells of the BBB is well documented [73]. Some studies showed an upregulation of the PDE4 enzyme after $A B I[74,75]$. Less is known about their role in cytoskeletal (CSK) function and their effects on cell adhesion molecules (CAMs). Given that CSK and CAMs are important for BBB function, there is a need to bridge this gap in our understanding. Initial findings indicating that PDE4 can mediate CAMs in peripheral cells might inform further mechanistic studies in endothelial cells of the BBB and potentially in neurons (Figure 4). Another effect of $A B I$ is an upregulation of PDE expression that compromises the effects of cAMP in cell functioning, as shown in different $A B I$ models $[26,76]$.

Together, these findings support the notion that PDE4 inhibitors could restore brain function during early and later $\mathrm{ABI}$ disease stages by a dual mechanism (Figure 4) $[77,78]$ ) involving their anti-inflammatory effects following modulation of different inflammation pathways [49]. In line with the earlier section on inflammation, an increase in CAMP levels by PDE4 inhibition could lead to a shift towards an anti-inflammatory state in various cells [68]. More specifically, PDE4B, but not PDE4D, appears to have a crucial role in the lipopolysaccharide (LPS)-induced inflammatory response $[79,80]$, indicating that this PDE4 subtype has a crucial role in microglia activation. This could be interesting for early as well as later stages of $A B I$. A second mechanism for the restoration of brain function after $A B I$ is enhancement of neuroplasticity following modulation of the CAMP/PKA/CREB plasticity pathway [50]. This might be most relevant during later stages of ABI.

With respect to the effects of PDE4 inhibitors on neuronal plasticity, including increased BDNF levels [29] and AMPA receptor upregulation [81], various studies suggest that enhanced neuronal plasticity contributes to the positive effects of different PDE4 inhibitors on different cognitive functions in 


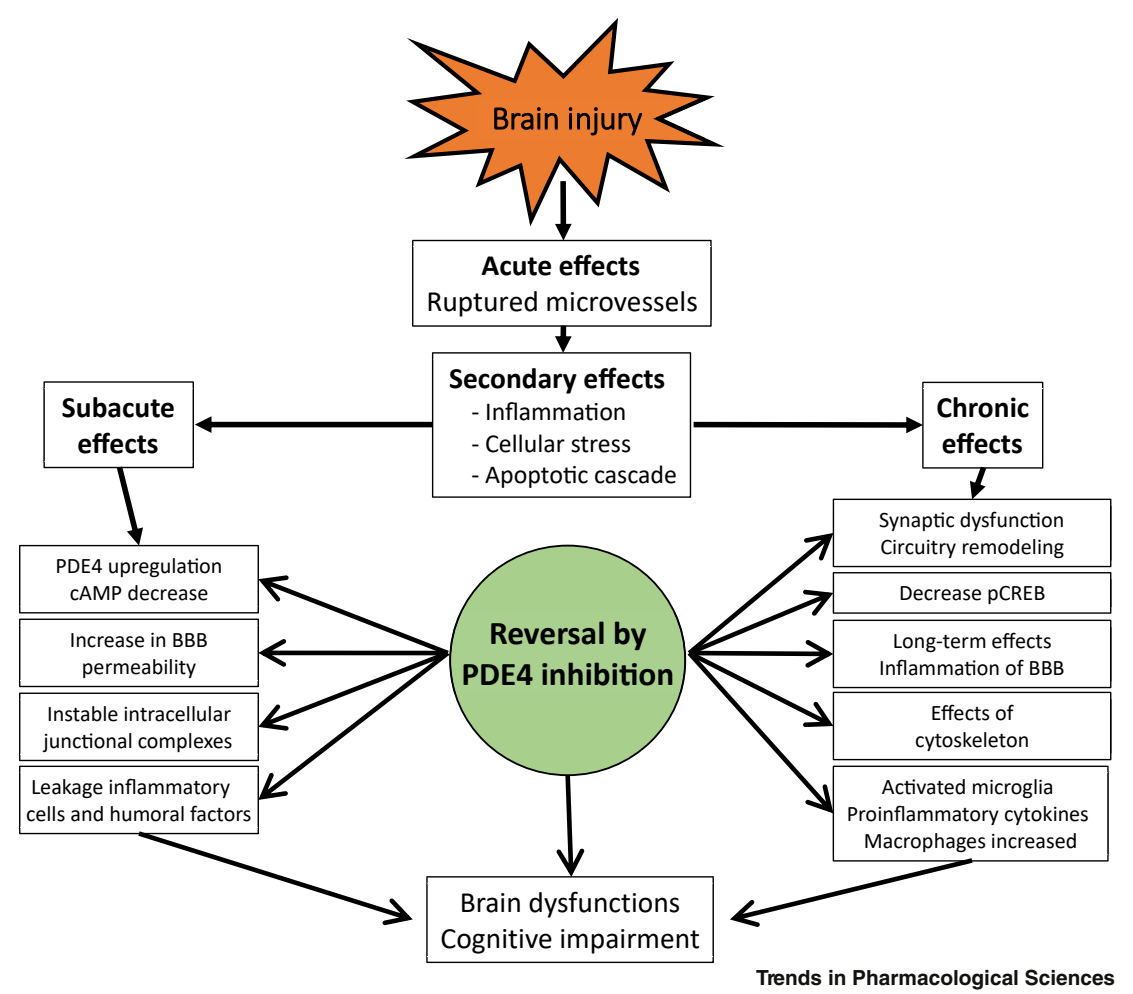

Figure 4. A Central Role for Phosphodiesterase 4 (PDE4) Inhibition in Acquired Brain Injury (ABI).

Molecular mechanisms: in a fluid percussion injury (FPI) model, PDE4 enzymes were upregulated and for certain subtypes this correlated with a decrease in CAMP levels and protein kinase A (PKA) activation in the hippocampus. In a four-vessel occlusion model, this increase in PDE4 enzyme levels was attenuated by rolipram. Hippocampal phosphorylated cAMP response element-binding protein ( $\mathrm{PCREB}$ ) levels were reduced in the FPI model and the PDE4B inhibitor A33 normalized these levels at 13 weeks after ABI. Cellular mechanisms: the sustained increase in blood-brain barrier (BBB) permeability and leakage of inflammatory cells and humoral factors aggravate BBB dysfunction. The PDE4 inhibitor, BBB022A, decreased damage to the BBB in a transient middle cerebral artery occlusion model. In a FPI model, increased tumor necrosis factor (TNF)- $\alpha$ levels were decreased by $\mathrm{A} 33$ at 3 months. Rolipram reduced levels of interleukin (IL)- $1 \beta$ and TNF- $\alpha$. Subcellular mechanisms: most studies with PDE4 inhibitors were done in peripheral cells, where they inhibit the expression of a variety of cell adhesion molecules (CAMs). The Rho pathway is an integrin-mediated signaling pathway. In an assay for the formation of integrin-dependent adhesive structures in rat embryo fibroblasts, rolipram suppressed RhoA activity, possibly relieving contractile forces and allowing the cytoskeletal rearrangement required for integrin complex assembly. Behavioral mechanisms: most cognition studies in $A B I$ models used rolipram, which was efficacious in, among others, a spatial Morris swim task and fear-conditioning tasks. The PDE4B inhibitor A33 improved long-term spatial memory retention and spatial working memory deficits by FPI in a Morris water maze and impairments in cue and contextual fear conditioning by moderate FPI.

different ABI models. Interestingly, this has been shown for PDE4B inhibitors (e.g., [82]) as well as for PDE4D inhibitors (e.g., [83]). The relative contribution of anti-inflammatory effects and enhanced neuroplasticity to the effects of PDE4 inhibitors on cognition is not yet fully understood. However, there is substantial evidence that microglia function is directly related to neuroplasticity, and that these might go hand in hand during different phases of brain damage [84].

In conclusion, PDE4 inhibitors could represent a novel class of drugs for the treatment of residual symptoms in $A B I$, attenuating the pathophysiological consequences via their anti-inflammatory effects and their positive effects on neuroplasticity. Several animal studies have shown promising 
effects of PDE4 inhibitors on the functional outcome after ABI (Figure 4). The finding that PDE4 inhibition was still effective when treatment started 3 months after the induction of brain trauma also appears promising for clinical applications [85]. Thus, clinical studies are indicated to demonstrate the potential of PDE4 inhibitors after stroke and brain trauma.

\section{Miscellaneous Diseases}

There are other CNS indications for which PDE4 could be relevant. There are some neurodevelopment diseases in which PDE4 inhibition has positive effects, such as fragile $X$ syndrome [34], Rubinstein-Taybi syndrome [35], juvenile Batten disease [36], and Rett syndrome [37]. Using genetic animal models, these studies showed that PDE4 inhibitors improved brain-related parameters that were typical for each disease. In addition, PDE4 inhibition restored cognitive functions in these different disease models. These studies suggested that the effects were related to restoring cAMP function in development and could also be linked to increased neuroplasticity after PDE4 inhibition. There is also good support for the notion that PDE4B (but not PDE4D) could be relevant for the treatment of depression [56]. These various studies show a pleiotropic effect of PDE4 inhibitors. This might be related to the central role of CAMP in different crucial cell functions and suggest that PDE4 inhibitors can regulate these disturbed processes in different disease states.

\section{Strategies Towards Safer and More Selective PDE4 Inhibition}

As mentioned earlier, clinical development of PDE4 inhibitors has been hampered by severe adverse effects, including nausea, emesis, and diarrhea. Selective inhibition of PDE4 subtypes (e.g., PDE4B) or isoforms (e.g., PDE4D1) could provide a more promising strategy to reduce the adverse effects and improve the therapeutic index of such inhibitors. However, this might be challenging given that all PDE4 genes show large homology, especially PDE4B and PDE4D, and produce highly similar catalytic domains. Nevertheless, the PDE4 subtypes exhibit subtle differences in protein structure, which have enabled the development of PDE4 subtype-specific inhibitors (Figure 5) $[86,87]$ ). Although subtypespecific inhibition is possible through interactions with nonconserved residues, adverse effects can still arise. Notably, expression and gene deletion studies revealed that PDE4D is the main mediator of emetic effects [88], suggesting that inhibition of other PDE4 subtypes would result in safer pharmacological profiles. Although this might be interesting for indications in which PDE4B appears to be relevant, it poses a challenge for the generation of procognitive effects, which appear to be related to PDE4D [56].

In addition to protein sequence differences among PDE4 subtypes that can confer inhibitor selectivity, PDE4 naturally exhibits different conformations showing distinct affinities for its prototypic inhibitor rolipram: the high-affinity rolipram-binding site (HARBS) and low-affinity rolipram-binding site (LARBS) [89]. Although the exact nature of HARBS and LARBS is unknown, prior studies indicated that specific cellular functions are regulated by either HARBS or LARBS conformers [90]. Given that HARBS occupancy correlates with emetic responses [91], it is hypothesized that inhibition of LARBS could reduce these effects [92]. HARBS depends on interactions with the UCR2 domain, and dimerized (i.e., long) isoforms stabilize the enzyme in the HARBS conformation [93]. However, neither dimerization nor the presence of UCR1 are requirements for HARBS, suggesting that short isoforms, which do not dimerize, can also exhibit HARBS [89]. Post-translational modifications (e.g., PKA phosphorylation) and interactions with partner proteins (e.g., XAP2), which have divergent effects on enzyme activity, can all increase rolipram sensitivity (e.g., [94]). Similarly, the affinity of the UCR2-interacting PDE4D inhibitor BPN14770 was increased in PDE4D constructs with mutations mimicking PKA phosphorylation [52]. It is proposed that PKA phosphorylation disrupts the UCR1-UCR2 module and that, in dimers, the UCR2 of one molecule can be 'trans-capped' onto the catalytic domain of the other, providing additional UCR2-inhibitor interactions $[95,96]$. This implies that PKA activation 'liberates' the UCR module to facilitate both CAMP hydrolysis and inhibitor binding, reflected by enzyme activation and increased inhibitor affinity, respectively. Interestingly, the PDE4D-selective and UCR2-interacting inhibitors PMNPQ and RS25344 [95] and those from the GEBR family shows similar affinities towards short and long PDE4D forms [97,98], suggesting that UCR2-inhibitor interactions also occur in monomeric PDE4. Additionally, interactions of the $C$ terminus with UCR2 and PMNPQ have been 
Long

PDE $4 A-4 / 5 / 8 / 10 / 11$

PDE $4 B-1 / 3 / 4$

PDE $4 C-1 / 2 / 3$

PDE4D - 3/4/5/7/8/9

Short

PDE4B - 2

PDE4D - 1

Supershort

PDE $4 A-1$

PDE4B - 5

PDE4D $-2 / 6$

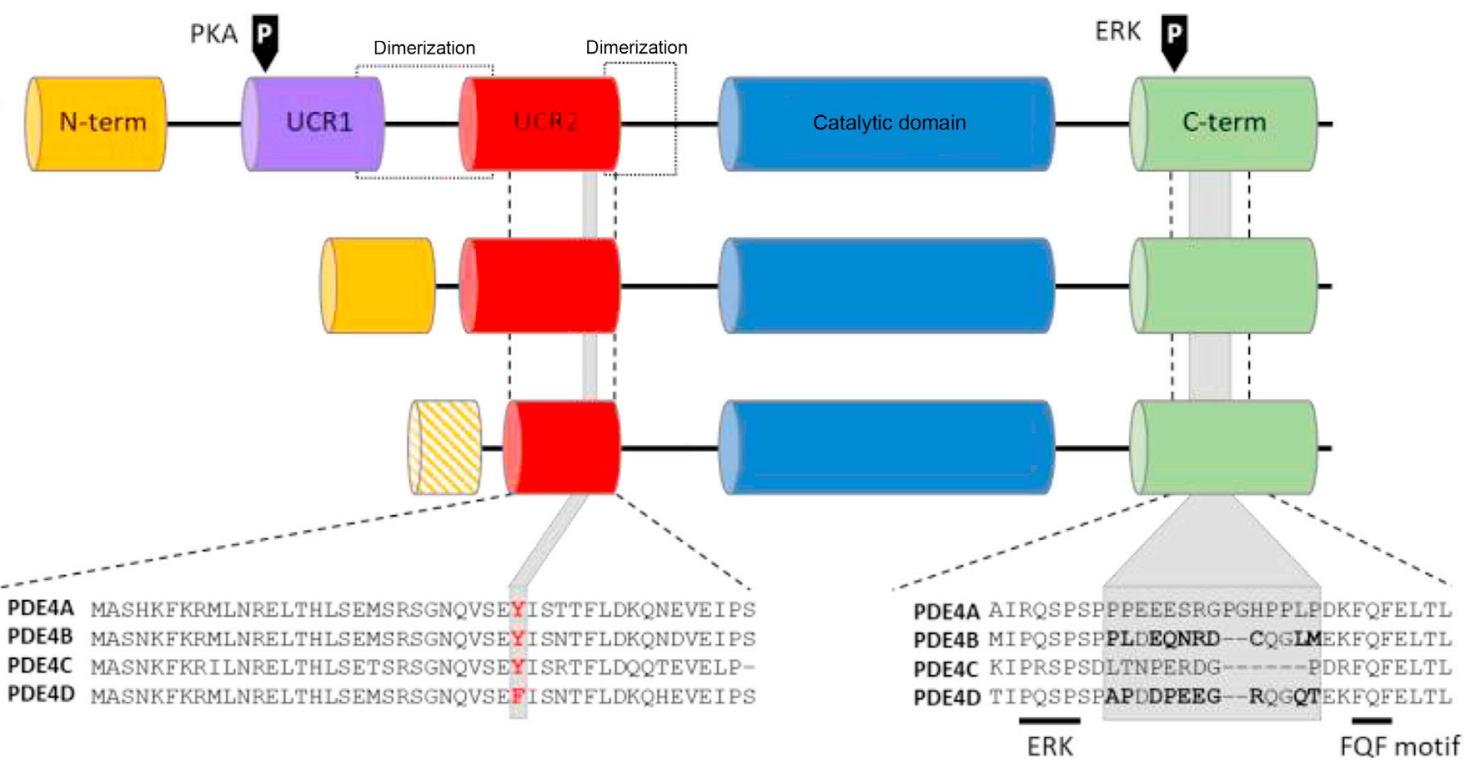

Trends in Pharmacological Sciences

Figure 5. Protein Domain Organization of Phosphodiesterase 4 (PDE4) Subtypes Categorizing Long, Short, and Supershort Isoforms.

Global organization of regulatory domains is conserved among PDE4 subtypes (i.e., PDE4A-D) with isoform-specific N-termini, upstream conserved regions (UCR1 and UCR2), a catalytic domain, and a C terminus. Protein isoforms can be categorized based on the presence of UCR1 and UCR2; long isoforms have both regulatory domains, short isoforms only contain UCR2, whereas supershort isoforms have a truncated UCR2. Some supershort forms have a unique N terminus (i.e., PDE4A1, PDE4B5, and PDE4D6), whereas others do not (i.e., PDE4D2). Additional regulatory residues are conserved across subtypes [e.g., phosphorylation sites for protein kinase A (PKA) causing enzyme activation and extracellular receptor kinase (ERK) causing long isoform inhibition and supershort activation, a FQF motif on the $\mathrm{C}$ terminus that enables interaction with partner proteins, and the dimerization domains that allow long isoforms to dimerize]. Despite this homology, the UCR2 and C terminus both contain nonconserved amino acids with which inhibitors can interact to generate selectivity towards a PDE4 subtype. In primates, PDE4D contains a phenylalanine residue in UCR2, whereas other PDE4 subtypes express a tyrosine (highlighted in red). This distinction has enabled the development of PDE4D-selective (e.g., BPN14770/D159797, PMNPQ, RS25344, and GEBR32a) and PDE4D-sparing (e.g., ABI-4/PF-06266047) inhibitors. Similarly, subtype-specific residues in the C terminus (indicated in bold) enable selective inhibitor binding to PDE4B (e.g., A-33 and a tetrahydrothiophene inhibitor).

observed that could provide additional effects on the binding affinity of UCR2-interacting inhibitors [95]. By contrast, subtype-specific residues in the $C$ terminus enable selective inhibition of PDE4B (e.g., A-33 [99] and a tetrahydrothiophene inhibitor [100]).

These findings indicate that HARBS and LARBS cannot be fully attributed to differences between long and short isoforms but rather result from the complex interplay of dimerization, protein-protein interactions, and post-translational modifications generating multiple conformations with different affinities (recently reviewed in [1]). Accordingly, inhibitors might preferentially bind isoforms bound to a partner protein or those that are post-translationally modified. PDE4 can be post-translationally modified in many ways [101] and, mainly through common UCR2 and C-terminus domains, can bind multiple partner proteins [102]. These effects can even be isoform specific via unique N-terminal domains (e.g., inhibition of PDE4D7 upon PKA phosphorylation [103] and preferential binding of $\beta$-arrestin to PDE4D5 [104]). Thus, although the regulation of the conformational state of PDE4 is complex, it can yield distinct inhibitor affinities, thereby offering the opportunity to target PDE4 isoforms or conformations specifically. Alternatively, PDE4 activity can be modulated using protein-protein interaction disruptors or compounds that act allosterically $[105,106]$.

Taken together, many factors influence the conformational state of PDE4 and, thus, inhibitor affinity. Prior studies have already shown that different modes of inhibition (i.e., solely through interactions with the catalytic domain or additional binding with UCR2) produce different cellular effects 
(e.g., [107]). Therefore, future studies should investigate what PDE4 subtypes or isoforms, and in what configuration, are involved in processes leading to adverse effects. Subsequently, inhibitors showing low affinity to these isoforms or configurations would produce safer pharmacological profiles. In addition, elucidating which isoforms, in which configuration, are involved in the processes leading to therapeutic activity will facilitate the development of more efficacious PDE4 inhibitors.

\section{Concluding Remarks and Future Perspectives}

The current overview provides a strong case for PDE4 as a potential target for different CNS diseases. Although the adverse effects of PDE4 inhibitors are a major issue, alternative ways are emerging to increase the therapeutic window for PDE4 inhibitors (see Outstanding Questions). A first approach may be linked to the properties of roflumilast. This is a nonselective PDE4 inhibitor that improves memory without any clear adverse effects $[39,40]$. Even for COPD, where three to five times higher doses are required, adverse effects are modest [7]. Further studies that investigate the binding properties of roflumilast at the PDE4 enzyme could reveal interesting characteristics and opportunities to improve the therapeutic window of PDE4 inhibitors. A second approach is to design PDE4 subtype- or isoform-selective inhibitors that have a more favorable therapeutic window. Linked to this, a better understanding of how the conformational state of PDE4 subtypes and isoforms is affected by different modulators (e.g., protein-protein interactions and phosphorylation) could further improve the clinical potential of these drugs.

Inhibition of PDE4 restores compromised cAMP functioning and reverses functional deficits in animal models of CNS disorders. Thus, it appears that neuroplasticity and anti-inflammatory effects are the key properties by which the effects of PDE4 inhibitors are mediated. These effects could also work synergistically (e.g., ABI). Based on the effects of PDE4 inhibitors on brain function in animal models and humans, and our current knowledge of the molecular biology of PDE4 subtypes, we believe that it is feasible to look for more efficacious and safe PDE4 inhibitors for the treatment of CNS diseases.

\section{Outstanding Questions}

How can we dissociate the positive clinical and adverse effects of PDE4 inhibitors?

Do we need isoform-selective PDE4 inhibitors to treat different CNS diseases?

Will the current structural biology approach be successful in finding selective ligands, given that the highly conserved catalytic domain of the four PDE4 isotypes makes it difficult to develop selective inhibitors?

How can we target specific pathways underlying different $\mathrm{CNS}$ diseases with PDE4 inhibitors?

How can we translate knowledge about the compartmentalization of PDE4 isoforms into drug discovery? Which other therapeutic strategies can be used, beyond direct PDE4 inhibition, to increase cAMP?

Could specific PDE4 inhibition also be effective in modulating neuro-

inflammatory diseases, such as AD?

\section{Resources}

ihttps://clinicaltrials.gov

\section{References}

1. Baillie, G.S. et al. (2019) Therapeutic targeting of 3',5'-cyclic nucleotide phosphodiesterases: inhibition and beyond. Nat. Rev. Drug Discov. 18, 770-796

2. Senft, G. et al. (1968) Influence of hydrochlorothiazide and other sulfamoyl diuretics on the activity of $3^{\prime}, 5^{\prime}$-AMP phosphodiesterase in rat kidney. Naunyn. Schmiedebergs Arch. Exp. Pathol. Pharmakol. 259, 344-359 (in German)

3. Beavo, J.A. et al. (1970) Effects of xanthine derivatives on lipolysis and on adenosine $3^{\prime}, 5^{\prime}$ monophosphate phosphodiesterase activity. Mol. Pharmacol. 6, 597-603

4. Maurice, D.H. et al. (2014) Advances in targeting cyclic nucleotide phosphodiesterases. Nat. Rev. Drug Discov. 13, 290-314

5. Lakics, V et al. (2010) Quantitative comparison of phosphodiesterase mRNA distribution in human brain and peripheral tissues. Neuropharmacology 59, 367-374

6. Soderling, S.H. and Beavo, J.A. (2000) Regulation of CAMP and cGMP signaling: new phosphodiesterases and new functions. Curr. Opin. Cell Biol. 12, 174-179

7. Zuo, H. et al. (2019) Phosphodiesterases as therapeutic targets for respiratory diseases. Pharmacol. Ther. 197, 225-242

8. Pincelli, C. et al. (2018) Mechanisms underlying the clinical effects of apremilast for psoriasis. J. Drugs Dermatol. 17, 835-840
9. Kloner, R.A. et al. (2018) Cardiovascular safety of phosphodiesterase type 5 inhibitors after nearly 2 decades on the market. Sex Med. Rev. 6, 583-594

10. Bedenis, R. et al. (2014) Cilostazol for intermittent claudication. Cochrane Database Syst. Rev. 2014, CD003748

11. Prickaerts, J. et al. (2017) Investigational phosphodiesterase inhibitors in phase I and phase II clinical trials for Alzheimer's disease. Expert Opin. Investig. Drugs 26, 1033-1048

12. Geerts, H. et al. (2017) Phosphodiesterase 10 inhibitors in clinical development for CNS disorders. Expert Rev. Neurother. 17, 553-560

13. Heckman, P.R.A. et al. (2018) Phosphodiesterase inhibition and modulation of corticostriatal and hippocampal circuits: clinical overview and translational considerations. Neurosci. Biobehav. Rev. 87, 233-254

14. Strick, C.A. et al. (2010) Alterations in gene regulation following inhibition of the striatumenriched phosphodiesterase, PDE10A. Neuropharmacology. 58, 444-451

15. Padovan-Neto, F.E. et al. (2015) Facilitation of corticostriatal transmission following pharmacological inhibition of striatal phosphodiesterase 10A: role of nitric oxide-soluble guanylyl cyclase-cGMP signaling pathways. J. Neurosci. 35, 5781-5791

16. Ooms, M. et al. (2016) [18F]JNJ42259152 binding to phosphodiesterase 10A, a key regulator of medium 
spiny neuron excitability, is altered in the presence of cyclic AMP. J. Neurochem. 139, 897-906

17. Song, R.S. et al. (2016) Cross-regulation of phosphodiesterase 1 and phosphodiesterase 2 activities controls dopamine-mediated striatal alpha-amino-3-hydroxy-5-methyl-4isoxazolepropionic acid (AMPA) receptor trafficking. J. Biol. Chem. 291, 23257-23267

18. Seeger, T.F. et al. (2003) Immunohistochemica localization of PDE10A in the rat brain. Brain Res. 985, 113-126

19. Wang, P. et al. (2003) Identification and characterization of a new human type 9 cGMPspecific phosphodiesterase splice variant (PDE9A5) differential tissue distribution and subcellular localization of PDE9A variants. Gene 314, 15-27

20. Kotera, J. et al. (2004) Subcellular localization of cyclic nucleotide phosphodiesterase type 10A variants, and alteration of the localization by CAMPdependent protein kinase-dependent phosphorylation. J. Biol. Chem. 279, 4366-4375

21. van Duinen, M.V. et al. (2015) Treatment of cognitive impairment in schizophrenia: potential value of phosphodiesterase inhibitors in prefrontal dysfunction. Curr. Pharm. Des. 21, 3813-3828

22. Dyck, B. et al. (2017) Discovery of selective phosphodiesterase 1 inhibitors with memory enhancing properties. J. Med. Chem. 60, 3472-3483

23. Reneerkens, O.A. et al. (2009) Selective phosphodiesterase inhibitors: a promising target for cognition enhancement. Psychopharmacology (Berl) 202, 419-443

24. Wennogle, L.P. et al. (2017) Phosphodiesterase 1: a unique drug target for degenerative diseases and cognitive dysfunction. Adv. Neurobiol. 17, 349-384

25. Zhang, C. et al. (2017) The role of phosphodiesterase- 2 in psychiatric and neurodegenerative disorders. Adv. Neurobiol. 17, 307-347

26. Yasmeen, S. et al. (2019) Cyclic nucleotide phosphodiesterases (PDEs) and endothelial function in ischaemic stroke. A review. Cell Signal. 61, 108-119

27. Noma, K. and Higashi, Y. (2018) Cilostazol for treatment of cerebral infarction. Expert Opin. Pharmacother. 19, 1719-1726

28. Yanai, S. and Endo, S. (2019) PDE3 inhibitors repurposed as treatments for age-related cognitive impairment. Mol. Neurobiol. 56, 4306-4316

29. Wang, H. et al. (2018) Targeting phosphodiesterase 4 as a potential therapeutic strategy for enhancing neuroplasticity following ischemic stroke. Int. J. Biol. Sci. 14, 1745-1754

30. Bolger, G.B. (2017) The PDE4 cAMP-specific phosphodiesterases: targets for drugs with antidepressant and memory-enhancing action. Adv. Neurobiol. 17, 63-102

31. Heckman, P.R. et al. (2016) Phosphodiesterase inhibition and regulation of dopaminergic frontal and striatal functioning: clinical implications. Int. J. Neuropsychopharmacol. 19, 1-16

32. Sakkas, L.l. et al. (2017) Phosphodiesterase 4 inhibitors in immune-mediated diseases: mode of action, clinical applications, current and future perspectives. Curr. Med. Chem. 24, 3054-3067

33. Medina-Rodriguez, E.M. et al. (2017) Promoting in vivo remyelination with small molecules: a neuroreparative pharmacological treatment for Multiple Sclerosis. Sci. Rep. 7, 43545

34. Gurney, M.E. et al. (2017) Multiple behavior phenotypes of the fragile-X syndrome mouse model respond to chronic inhibition of phosphodiesterase-4D (PDE4D). Sci. Rep. 7, 14653

35. Bourtchouladze, R. et al. (2003) A mouse model of Rubinstein-Taybi syndrome: defective long-term memory is ameliorated by inhibitors of

phosphodiesterase 4. Proc. Natl. Acad. Sci. U S A 100, 10518-10522

36. Aldrich, A. et al. (2016) Efficacy of phosphodiesterase-4 inhibitors in juvenile Batten disease (CLN3). Ann. Neurol. 80, 909-923

37. $\mathrm{Bu}, \mathrm{Q}$. et al. (2017) CREB signaling is involved in Rett syndrome pathogenesis. J. Neurosci. 37, 36713685

38. Zhang, H.T. (2009) Cyclic AMP-specific phosphodiesterase-4 as a target for the development of antidepressant drugs. Curr. Pharm. Des. 15, 1688-1698

39. Blokland, A. et al. (2019) Acute treatment with the PDE4 inhibitor roflumilast improves verbal word memory in healthy old individuals: a double-blind placebo-controlled study. Neurobiol. Aging 77, $37-43$

40. Gilleen, J. et al. (2018) An experimental medicine study of the phosphodiesterase-4 inhibitor, roflumilast, on working memory-related brain activity and episodic memory in schizophrenia patients. Psychopharmacology (Berl). Published online December 8, 2018. https://doi.org/10.1007/ s00213-018-5134-y.

41. Shim, Y.S. et al. (2014) Effects of daily low-dose treatment with phosphodiesterase type 5 inhibitor on cognition, depression, somatization and erectile function in patients with erectile dysfunction: a double-blind, placebo-controlled study. Int. J. Impot. Res. 26, 76-80

42. Reneerkens, O.A et al. (2013) The effects of the phosphodiesterase type 5 inhibitor vardenafil on cognitive performance in healthy adults: a behavioral-electroencephalography study. J. Psychopharmacol. 27, 600-608

43. Dorner-Ciossek, C. et al. (2017) Role of PDE9 in cognition. Adv. Neurobiol. 17, 231-254

44. Brown, D. et al. (2019) Evaluation of the efficacy, safety, and tolerability of BI 409306, a novel phosphodiesterase 9 inhibitor, in cognitive impairment in schizophrenia: a randomized, double-blind, placebo-controlled, Phase II trial. Schizophr. Bull. 45, 350-359

45. Whiteley, E.L. et al. (2019) PDE10A mutations help to unwrap the neurobiology of hyperkinetic disorders. Cell Signal. 60, 31-38

46. Jankowska, A. et al. (2019) Advances in discovery of PDE10A inhibitors for CNS-related disorders. Part 1: overview of the chemical and biological research. Curr. Drug Targets 20, 122-143

47. Zagorska, A et al. (2018) Phosphodiesterase 10 inhibitors - novel perspectives for psychiatric and neurodegenerative drug discovery. Curr. Med. Chem. 25, 3455-3481

48. Hegde, S. et al. (2016) Phosphodiesterase 11A (PDE11A), enriched in ventral hippocampus neurons, is required for consolidation of social but not nonsocial memories in mice. Neuropsychopharmacology 41, 2920-2931

49. Li, H. et al. (2018) Phosphodiesterase-4 inhibitors for the treatment of inflammatory diseases. Front. Pharmacol. 9, 1048

50. Kandel, E.R. (2012) The molecular biology of memory: CAMP, PKA, CRE, CREB-1, CREB-2, and CPEB. Mol. Brain 5, 14

51. Hoerndli, F.J. et al. (2015) Neuronal activity and CaMKII regulate kinesin-mediated transport of synaptic AMPARs. Neuron 86, 457-474

52. Zhang, C. et al. (2018) Memory enhancing effects of BPN14770, an allosteric inhibitor of phosphodiesterase-4D, in wild-type and humanized mice. Neuropsychopharmacology 43, 2299-2309

53. Bollen, E. et al. (2014) Improved long-term memory via enhancing CGMP-PKG signaling requires 
cAMP-PKA signaling. Neuropsychopharmacology 39, 2497-2505

54. Peng, S. et al. (2014) Effects of selective phosphodiesterases-4 inhibitors on learning and memory: a review of recent research. Cell Biochem. Biophys. 70, 83-85

55. Lamontagne, S. et al. (2001) Localization of phosphodiesterase-4 isoforms in the medulla and nodose ganglion of the squirrel monkey. Brain Res. 920, 84-96

56. Zhang, C. et al. (2017) Comparison of the pharmacological profiles of selective PDE4B and PDE4D inhibitors in the central nervous system. Sci. Rep. 7, 40115

57. Calabresi, P et al. (2007) Dopamine-mediated regulation of corticostriatal synaptic plasticity. Trends Neurosci. 30, 211-219

58. Song, R.S. et al. (2013) ERK regulation of phosphodiesterase 4 enhances dopaminestimulated AMPA receptor membrane insertion. Proc. Natl. Acad. Sci. U S A 110, 15437-15442

59. Nishi, A. et al. (2008) Distinct roles of PDE4 and PDE10A in the regulation of CAMP/PKA signaling in the striatum. J. Neurosci. 28, 10460-10471

60. Tanaka, M. et al. (2017) Aggregation of scaffolding protein DISC1 dysregulates phosphodiesterase 4 in Huntington's disease. J. Clin. Invest. 127, 1438-1450

61. Olsen, C.M. and Liu, Q.S. (2016) Phosphodiesterase 4 inhibitors and drugs of abuse: current knowledge and therapeutic opportunities. Front. Biol. (Beijing) 11, 376-386

62. Schepers, M. et al. (2019) Targeting phosphodiesterases (PDE)-towards a tailor-made approach in multiple sclerosis treatment. Front. Immunol. 10, 1727

63. Klein, M. and Bopp, T. (2016) Cyclic AMP represents a crucial component of Treg cell-mediated immune regulation. Front. Immunol. 7, 315

64. Santiago, A. et al. (2018) Roflumilast promotes memory recovery and attenuates white matter injury in aged rats subjected to chronic cerebral hypoperfusion. Neuropharmacology 138, 360-370

65. Peter, D. et al. (2007) Differential expression and function of phosphodiesterase 4 (PDE4) subtypes in human primary CD4+ T cells: predominant role of PDE4D. J. Immunol. 178, 4820-4831

66. Xuan, W. et al. (2015) The chemotaxis of M1 and M2 macrophages is regulated by different chemokines. J. Leukoc. Biol. 97, 61-69

67. Ghosh, M. et al. (2016) Cyclic AMP is a key regulator of $\mathrm{M} 1$ to $\mathrm{M} 2 \mathrm{a}$ phenotypic conversion of microglia in the presence of Th2 cytokines. J. Neuroinflammation. 13, 9

68. You, T. et al. (2017) Roflupram, a phosphodiesterase 4 inhibitor, suppresses inflammasome activation through autophagy in microglial cells. ACS Chem. Neurosci. 8, 2381-2392

69. Goodman, A.D. et al. (2016) Ibudilast for the treatment of multiple sclerosis. Expert Opin. Investig. Drugs 25, 1231-1237

70. Fox, R.J. et al. (2018) Phase 2 trial of ibudilast in progressive multiple sclerosis. N. Engl. J. Med. 379, 846-855

71. Johnson, V.E. et al. (2013) Inflammation and white matter degeneration persist for years after a single traumatic brain injury. Brain 136, 28-42

72. Prakash, R. and Carmichael, S.T. (2015) Blood-brain barrier breakdown and neovascularization processes after stroke and traumatic brain injury. Curr. Opin. Neurol. 28, 556-564

73. Rampersad, S.N. et al. (2010) Cyclic AMP phosphodiesterase 4D (PDE4D) tethers EPAC1 in a vascular endothelial cadherin (VE-Cad)-based signaling complex and controls CAMP-mediated vascular permeability. J. Biol. Chem. 285, 33614 33622

74. Wilson, N.M. et al. (2016) Traumatic brain injury upregulates phosphodiesterase expression in the hippocampus. Front. Syst. Neurosci. 10, 5

75. Li, L.X. et al. (2011) Prevention of cerebral ischemiainduced memory deficits by inhibition of phosphodiesterase-4 in rats. Metab. Brain Dis. 26, 37-47

76. He, Z. et al. (2012) Ischemia-induced increase in microvascular phosphodiesterase 4D expression in rat hippocampus associated with blood brain barrier permeability: effect of age. ACS Chem. Neurosci. 3, 428-432

77. Belayev, L. et al. (1998) Protection against blood-brain barrier disruption in focal cerebral ischemia by the type IV phosphodiesterase inhibitor BBB022: a quantitative study. Brain Res. 787. 277-285

78. Atkins, C.M. et al. (2009) Deficits in ERK and CREB activation in the hippocampus after traumatic brain injury. Neurosci. Lett. 459, 52-56

79. Jin, S.L. and Conti, M. (2002) Induction of the cyclic nucleotide phosphodiesterase PDE4B is essential for LPS-activated TNF-alpha responses. Proc. Natl. Acad. Sci. U S A 99, 7628-7633

80. Jin, S.L. et al. (2005) Specific role of phosphodiesterase 4B in lipopolysaccharideinduced signaling in mouse macrophages. J. Immunol. 175, 1523-1531

81. Vogel, E.W., 3rd et al. (2017) Phosphodiesterase-4 inhibition restored hippocampal long term potentiation after primary blast. Exp. Neurol. 293, 91-100

82. Wilson, N.M. et al. (2017) Therapeutic benefits of phosphodiesterase 4B inhibition after traumatic brain injury. PLoS One 12, e0178013

83. Titus, D.J. et al. (2018) A negative allosteric modulator of PDE4D enhances learning after traumatic brain injury. Neurobiol. Learn. Mem. 148, 38-49

84. Salter, M.W and Stevens, B. (2017) Microglia emerge as central players in brain disease. Nat. Med. 23, 1018-1027

85. Titus, D.J. et al. (2016) Chronic cognitive dysfunction after traumatic brain injury is improved with a phosphodiesterase 4B inhibitor. J. Neurosci. 36, 7095-7108

86. Richter, W. and Conti, M. (2002) Dimerization of the type 4 cAMP-specific phosphodiesterases is mediated by the upstream conserved regions (UCRs). J. Biol. Chem. 277, 40212-40221

87. Bolger, G.B. et al. (2015) Dimerization of cAMP phosphodiesterase-4 (PDE4) in living cells requires interfaces located in both the UCR1 and catalytic unit domains. Cell Signal. 27, 756-769

88. Mori, F. et al. (2010) The human area postrema and other nuclei related to the emetic reflex express cAMP phosphodiesterases 4B and 4D. J. Chem. Neuroanat. 40, 36-42

89. Jacobitz, S. et al. (1996) Mapping the functional domains of human recombinant phosphodiesterase 4A: structural requirements for catalytic activity and rolipram binding. Mol. Pharmacol. 50, 891-899

90. Boomkamp, S.D. et al. (2014) Epac and the high affinity rolipram binding conformer of PDE4 modulate neurite outgrowth and myelination using an in vitro spinal cord injury model. Br. J. Pharmacol. 171, 2385-2398

91. Hirose, R. et al. (2007) Correlation between emetic effect of phosphodiesterase 4 inhibitors and their occupation of the high-affinity rolipram binding site in Suncus murinus brain. Eur. J. Pharmacol. 573, 93-99 
92. Duplantier, A.J. et al. (1996) Biarylcarboxylic acids and-amides: inhibition of phosphodiesterase type IV versus $[3 \mathrm{H}]$ rolipram binding activity and their relationship to emetic behavior in the ferret. J. Med. Chem. 39, 120-125

93. Richter, W. and Conti, M. (2004) The oligomerization state determines regulatory properties and inhibitor sensitivity of type 4 cAMPspecific phosphodiesterases. J. Biol. Chem. 279 30338-30348

94. Bolger, G.B. et al. (2003) Attenuation of the activity of the CAMP-specific phosphodiesterase PDE4A5 by interaction with the immunophilin XAP2. J. Biol. Chem. 278, 33351-33363

95. Burgin, A.B. et al. (2010) Design of phosphodiesterase 4D (PDE4D) allosteric modulators for enhancing cognition with improved safety. Nat. Biotechnol. 28, 63-70

96. Cedervall, P. et al. (2015) Engineered stabilization and structural analysis of the autoinhibited conformation of PDE4. Proc. Natl. Acad. Sci. U S A 112, E1414-1422

97. Ricciarelli, R. et al. (2017) Memory-enhancing effects of GEBR-32a, a new PDE4D inhibitor holding promise for the treatment of Alzheimer's disease. Sci. Rep. 7, 46320

98. Prosdocimi, T. et al. (2018) Molecular bases of PDE4D inhibition by memory-enhancing GEBR library compounds. Biochemistry 57, 2876 2888

99. Fox, D., 3rd. et al. (2014) Structural basis for the design of selective phosphodiesterase 4B inhibitors. Cell Signal. 26, 657-663
100. Kranz, M. et al. (2009) Identification of PDE4B Over 4D subtype-selective inhibitors revealing an unprecedented binding mode. Bioorg. Med. Chem. 17, 5336-5341

101. Mika, D. and Conti, M. (2016) PDE4D phosphorylation: a coincidence detector integrating multiple signaling pathways. Cell Signal. 28, 719-724

102. McCahill, A.C. et al. (2008) PDE4 associates with different scaffolding proteins: modulating interactions as treatment for certain diseases. Handlb Exp. Pharmacol. 125-166

103. Byrne, A.M. et al. (2015) The activity of cAMPphosphodiesterase 4D7 (PDE4D7) is regulated by protein kinase A-dependent phosphorylation within its unique N-terminus. FEBS Lett. 589, 750-755

104. Bolger, G.B. et al. (2003) The unique amino-terminal region of the PDE4D5 cAMP phosphodiesterase isoform confers preferential interaction with betaarrestins. J. Biol. Chem. 278, 49230-49238

105. Omar, F. et al. (2019) Small-molecule allosteric activators of PDE4 long form cyclic AMP phosphodiesterases. Proc. Natl. Acad. Sci. U S A 116, 13320-13329

106. Lee, L.C. et al. (2013) Targeting protein-protein interactions within the cyclic AMP signaling system as a therapeutic strategy for cardiovascular disease. Future Med. Chem. 5, 451-464

107. Day, J.P. et al. (2011) Elucidation of a structural basis for the inhibitor-driven, p62 (SOSTM1)-dependent intracellular redistribution of CAMP phosphodiesterase-4A4 (PDE4A4). J. Med. Chem. 54, 3331-3347 